\title{
Safety and Efficacy of Camrelizumab Combined with Apatinib for Advanced Hepatocellular Carcinoma with Portal Vein Tumor Thrombus: A Multicenter Retrospective Study
}

This article was published in the following Dove Press journal: OncoTargets and Therapy

Guosheng Yuan, (D) ${ }^{1, *}$ Xiao Cheng, ${ }^{1, *}$ Qi Li, ${ }^{1,2}$ Mengya Zang,' Wei Huang, ${ }^{3}$ Wenzhe Fan, ${ }^{4}$ Tao Wu, ${ }^{5}$ Jian Ruan, ${ }^{6}$ Wencong Dai, 'Wenxuan Yu,' Mian Chen, ${ }^{7}$ Yabing Guo,' Xiaoyun $\mathrm{Hu}$,' Jinzhang Chen ${ }^{1,2}$

'Department of Infectious Diseases and Hepatology Unit, Nanfang Hospital, Southern Medical University, Guangzhou, People's Republic of China; ${ }^{2}$ Department of Oncology, Nanfang Hospital, Southern Medical University, Guangzhou, People's Republic of China; ${ }^{3}$ Department of Oncology, ShunDe Hospital, Southern Medical University, Foshan, People's Republic of China; ${ }^{4}$ Department of Interventional Oncology, The First Affiliated Hospital of Sun Yat-Sen University, Guangzhou, People's Republic of China; ${ }^{5}$ Department of Infectious Disease, Hainan General Hospital, Haikou, People's Republic of China; ${ }^{6}$ Department of Medical Oncology, The First Affiliated Hospital, The First Affiliated Hospital, School of Medicine, Zhejiang University, Hangzhou, People's Republic of China;

${ }^{7}$ Transplant Immunology Laboratory, Churchill Hospital, Oxford University Hospitals NHS Foundation Trust, Headington, Oxford, UK

*These authors contributed equally to this work
Introduction: Previous trials demonstrated that anti-angiogenesis or anti-programmed death protein 1 (PD-1) monotherapy showed unsatisfied effect in advanced hepatocellular carcinoma (HCC). No study existed that focus on the effects of camrelizumab and apatinib ("C+A") combination therapy for $\mathrm{HCC}$ patients with the location and extent of portal vein tumor thrombus (PVTT) as the main variable being assessed. This study was to compare the efficacy and tolerability of "C+A" for HCC patients with PVTT.

Methods: We retrospectively analyzed patients with advanced HCC and PVTT who underwent " $C+A$ " therapy in a multicenter retrospective cohort from Jan 2019 to July 2020. Outcomes of patients who underwent " $\mathrm{C}+\mathrm{A}$ " were analyzed by using the Kaplan-Meier method according to types of PVTT: PVTT in the main portal vein (type A), PVTT in the first-order portal vein branch (type B), and PVTT in second- or lower-order portal vein branches (type C).

Results: Sixty-three patients were finally included and the mean duration of follow-up was $12.6 \pm 4.5$ months. The objective response rate (ORR) and disease control rate (DCR) for the whole cohort were $44.0 \%$ and $75.0 \%$, respectively. The median overall survival (OS), progression-free survival (PFS) and time to progression (TTP) were 14.8 months, 11.8 months and not yet reached (NR), respectively. Patients with type B (OS, 15.9 months; PFS, 14.0 months; TTP, NR) or type C (OS, 16.0 months; PFS, 14.9 months; NR) PVTT appear to have better survival benefits compared with type A (OS, 5.8 months; PFS, 5.0 months; TTP, 7.0 months). Along with AFP, the absence of main PVTT was an independent predictive factor for survival at uni- and multivariate analysis.

Conclusion: Camrelizumab and apatinib yielded a promising outcome in patients with advanced $\mathrm{HCC}$ who developed a tumor thrombus in the first lower-order portal vein branches and was generally safe and had manageable side effects.

Keywords: hepatocellular carcinoma, HCC, camrelizumab, apatinib, overall survival, OS

\section{Introduction}

Hepatocellular carcinoma (HCC) is the second leading cause of cancer-related death in China, where HCC cases alone account for more than half of new cases and deaths related to HCC worldwide. ${ }^{1,2}$ Current recommendations for screening aim to identify smaller tumors that can be treated with resection, local-regional therapy or liver transplantation. $^{3-5}$ However, a large number of patients are beyond criteria that would be considered for curative approaches, whose survival rate is typically poor,
Correspondence: Jinzhang Chen; Xiaoyun

Department of Infectious Diseases and Hepatology Unit, Nanfang Hospital, Southern Medical University, 1838 North Guangzhou Ave, Guangzhou, Guangdong 510515, People's Republic of China

Tel + 8620-13802522545;

$+8620-15625010540$

Fax + 8620-62787430; + 8620-62787430

Email chenjinzhang@smu.edu.cn;

huxiaoyun143@I63.com 
especially for those with portal vein tumor thrombus (PVTT) (2-4 months if left untreated). ${ }^{6,7}$

In China, the incidences of PVTT have been reported to range from $44 \%$ to $62.2 \% .{ }^{8,9}$ The treatment of HCC patients with PVTT is based on the patients' liver function, the stage of hepatic lesion, and the extent of PVTT. For example, transcatheter arterial chemoembolization (TACE) is recommended as the primary treatment for PVTT patients with Child-Pugh A liver function, while its efficacy in these patients still remained unsatisfactory. ${ }^{9-13}$ Another proposed treatment option is molecular-targeted drug, such as Sorafenib, with a reported median survival time as short as 10.7 months. ${ }^{14,15}$ Therefore, more effective treatment strategies are urgently needed for this part of patients.

Immune checkpoint inhibitor (ICI) therapy, particularly antibodies targeting the programmed cell death-1 (PD-1)/ programmed cell death ligand-1 (PD-L1) pathway, has now been the backbone of numerous combination regimens aimed at improving the tumor response and survival of HCC patients. ${ }^{16-19}$ However, it was not until Mid-2018 that two anti-PD-1 antibodies, nivolumab and pembrolizumab, received approval as second-line drugs for HCC in China. Moreover, the high price (about 63 thousand USD a year vs 87 thousand USD a year, respectively) limited their accessibility. Fortunately, camrelizumab, one of the domestically developed anti-PD-1 antibodies approved in China, has a notable price advantage (17.0 thousand USD a year) and was widely applied for cancer therapy since its approval. According to the randomized, multicenter Phase 2/3 trial (NCT02989922) of camrelizumab (SHR-1210) in 217 patients with advanced HCC, the objective response rate (ORR) was $13.8 \%{ }^{20}$ The above study indicates the necessity of exploring combinational strategies with other treatments to enhance the efficacy of camrelizumab.

The most extensively tested combination regimen for advanced HCC comprises anti-PD1/anti-PDL1 plus antiangiogenic agents. Recently, a study on anti-PD-L1 antibody and vascular endothelial growth factor (VEGF)-antibody combination (atezolizumab and bevacizumab), has shown superior overall survival (OS) and progression-free survival (PFS) compared to sorafenib in patients with advanced HCC, indicating antiangiogenic therapies may enhance the response to PD-1/PD-L1 blockade and improve survival. ${ }^{21,22}$ Apatinib, a selective inhibitor of vascular endothelial growth factor receptor (VEGFR)-2, has demonstrated antitumoral activity in HCC when combined with TACE in several studies. ${ }^{23-25}$ Moreover, a Phase I trial of patients with advanced HCC and gastric cancer has demonstrated that camrelizumab and apatinib combination therapy was effective and well tolerated. ${ }^{26}$ Here, we report the safety and efficacy of camrelizumab in combination with apatinib in a multi-center treatment cohort of HBV associated HCC patients with PVTT in China, aiming to reflect the treatment reality in HCC outside of clinical trial programs and to share our experience for treating advanced HCC with a different type of PVTT.

\section{Materials and Methods \\ Patients}

We retrospectively reviewed the medical records of patients with chronic hepatitis B (CHB) associated HCC and PVTT who underwent camrelizumab combined with apatinib (" $\mathrm{C}$ +A") therapy from Jan 2019 to July 2020 in 5 institutions: 1 . Nanfang Hospital, Southern Medical University, 2. Shunde Hospital, Southern Medical University, 3. The First Affiliated Hospital of Sun Yat-Sen University, 4. The First Affiliated Hospital, School of Medicine, Zhejiang University and 5. Hainan General Hospital, Haikou.

This study was designed and performed according to the Helsinki Declaration and was approved by the Medical Ethics Committee of every participating hospitals in this study. Written informed consent was obtained from each patient to retroactively review and report on their medical records.

\section{Inclusion and Exclusion Criteria}

Inclusion criteria: (1) patients with HCC diagnosed by two imaging modalities, or biopsy; (2) patients who were in stage $\mathrm{C}$ according to the Barcelona Clinic Liver Cancer (BCLC) staging system, ${ }^{27}$ and could not tolerate or refused surgery, radiation or ablation; (3) patients without heart, lung or kidney dysfunction; (4) patients with Child-Pugh A or B liver function; (5) presence of PVTT on 3-phase dynamic CT images obtained within 7 days before treatment.

Patients were excluded from this study if they (1) had undergone local-regional therapies, or liver transplantation during follow-up; (2) history of apatinib or any other PDL1/PD-1 antagonist treatment; (3) brain or leptomeningeal metastasis or uncontrolled medical disorders that could jeopardize the outcomes of the study; (4) women who were pregnant or breast feeding; (5) currently had or had a history of malignant tumors in addition to HCC; (6) positive $\mathrm{HAV} / \mathrm{HCV} / \mathrm{HDV} / \mathrm{HIV}$ serology.

\section{Classification of PVTT}

The criteria for PVTT classification was used as before $^{11,24,25,28-30}$ and the types of PVTT were classified 
into three subgroups: (a) type A was defined as PVTT in the main portal vein; (b) type B was defined as PVTT in the first-order portal vein branch (the right or left portal vein); and (c) type C was defined as PVTT in the secondor lower-order portal vein branches (segmental branches of portal vein or higher).

\section{Procedures}

\section{Dosage of Camrelizumab}

Camrelizumab was given at a fixed dose of $200 \mathrm{mg}$ every 3 weeks (q3w) intravenously.

\section{Administration Method of Apatinib}

Patients treated with apatinib received therapy at a daily metronomic oral dosage of $250 \mathrm{mg}$. Grade $3 / 4$ adverse events (AEs) led to dose modification (125 mg daily) or temporary interruption until symptoms resolved to grades $\leq 2$. Treatment continued until disease progression as defined by mRECIST, clinical progression (defined as an ECOG performance score $=4$ or symptomatic deterioration, including increased liver function tests), unacceptable toxicity, withdrawal of consent by the patient, a decision by the treating physician that discontinuation would be in the patient's best interest, or death. Patient was permitted to continue beyond progression if the investigator judged that the patient would benefit.

\section{Clinical and Laboratory Evaluation}

Clinical and laboratory data were collected from all patients prior to " $\mathrm{C}+\mathrm{A}$ " therapy. Clinical data included age, gender, BCLC stage, ECOG performance and ChildPugh score. In addition, imaging data were collected based on abdominal computed tomography (CT) and/or magnetic resonance imaging (MRI), including tumor size, number, vascular invasion, and extrahepatic metastasis. Laboratory data included alpha-fetoprotein (AFP), alanine aminotransferase (ALT), aspartate aminotransferase (AST), albumin, total bilirubin (TBIL), albumin (ALB), white blood cell (WBC), platelet (PLT), prothrombin time (PT), hepatitis B surface antigen ( $\mathrm{HBsAg}$ ), and hepatitis $\mathrm{B}$ e antigen (HBeAg).

The patients underwent CT or MRI at baseline, 6 weeks after treatment initiation, and about every 3 months thereafter. Efficacy of treatment was measured by overall survival (OS) (defined as the interval between the first dose of PD-1 administration until death or the last followup (the last time when patient's clinical data was recorded before July 31, 2020)), time to tumor progression (TTP) (defined as the time from first checkpoint inhibitor administration until the date that tumor progression was confirmed radiologically), and progression-free survival (PFS) (defined as the time from first checkpoint inhibitor administration until radiological disease progression or death, whatever came first). Treatment-related adverse events (TRAE) were recorded at every visit according to the US National Cancer Institute (NCI) Common Terminology Criteria for Adverse Events (CTCAE v4.03).

Tumor responses were evaluated according to the modified response evaluation criteria in solid tumor (mRECIST): ${ }^{31}$ (1) Complete response (CR), all enhanced imaging of the target lesions in the arterial phase disappeared; (2) Partial response (PR), the total reduction of the diameter of the target lesions (enhanced arterial phase) was $\geq 30 \%$; (3) Stable disease (SD), the diameter of the target lesion did not reduce to that in PR and did not increase to that in disease progression (PD); (4) Progressive disease (PD), the diameter of the target lesion (enhanced imaging in the arterial phase) increased by at least $20 \%$ compared with the baseline value, or new lesions appeared.

\section{Statistical Analysis}

All statistical analyses were performed using SPSS Statistics for Windows, Version 25.0 (SPSS Inc., Chicago, IL, USA). Data were expressed as counts and percentages for categorical variables and as Mean \pm standard deviation (SD) for continuous variables. Survival curves were calculated by using Kaplan-Meier methods. Univariate analyses were performed with the Log rank test. Variables with a $P$ value of less than 0.1 at univariate analysis were entered into a multivariate analysis. Multivariate analyses were performed with a Cox's proportional hazard regression model. All statistical analyses were based on 2-tailed hypothesis tests with a significance level of $P<0.05$.

\section{Results \\ Clinical Characteristics}

Between January 2019 and July 2020, 94 patients with CHBassociated advanced $\mathrm{HCC}$ and PVTT received a combination treatment of camrelizumab and apatinib ("C+A"), of which 63 were included in the final analyses (Figure 1). Thirty-one patients were excluded because of attending other clinical trials $(\mathrm{n}=2)$, accepting locoregional therapy during follow-up $(n=8)$, incomplete data $(n=13)$ or follow-up shorter than 6 weeks $(n=8)$. The demographic and laboratory characteristics at baseline are shown in Table 1 and the mean duration of 


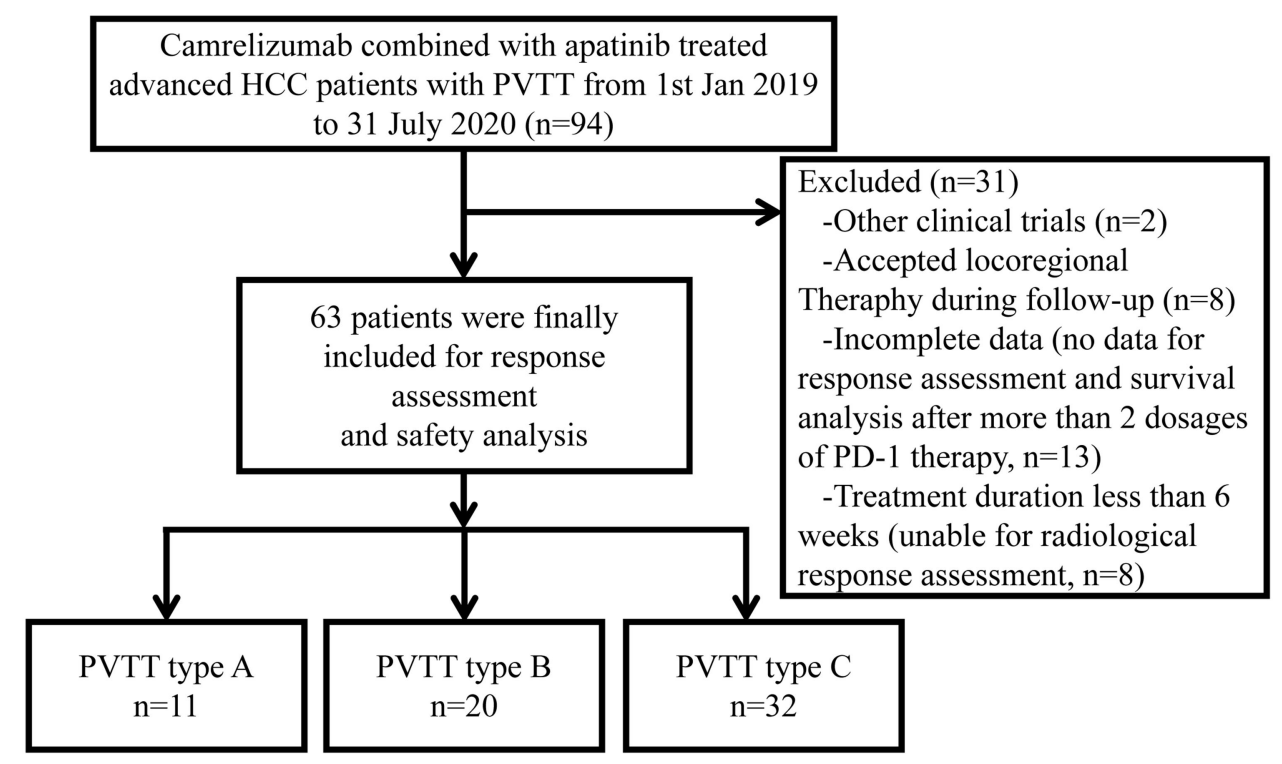

Figure I Patient recruitment flowchart.

follow-up was $12.6 \pm 4.5$ months. The mean age was $48.7 \pm$ 10.5 years, predominantly men $(92.1 \%)$ and $85.7 \%$ of patients were in CP A. All included patients are HBV infected and have received anti-viral treatment before " $\mathrm{C}+\mathrm{A}$ " initiation. Accordingly, they were divided into 3 groups: the PVTT type A group ( $\mathrm{n}=11$ patients; 9 males) and type $\mathrm{B}$ group $(n=20 ; 10$ males) and type $C$ group $(n=32 ; 30$ males). There were no differences in baseline characteristics among patients with different types of PVTT (Supplementary table 1).

\section{Tumor Response}

Tumor responses in patients with different types of PVTT are shown in Table 2. For all included patients, 1 had complete response (CR), 27 patients achieved partial response (PR), and 19 patients had stable disease (SD), resulting in an objective response rate (ORR) of $44.0 \%$ and disease control rate (DCR) of 75.0\%. Subgroup analysis revealed that DCR in patients with type B or C PVTT were $95.0 \%$ and $68.8 \%$, respectively, which were significantly higher than that of type A PVTT $(\chi 2=7.306, P=$ 0.026). However, the ORR among the three groups was not significantly different $(\mathrm{ORR}=27.3 \%, 50.0 \%$ and $46.9 \%$, respectively, for PVTT type A, B and C; $\chi^{2}=$ 1.640, $P=0.440$ ).

\section{PFS in Patients with Different Types of PVTT}

Median PFS was 11.8 months (95\% CI: 6.4, 17.2) for all patients (Figure 2A and Table 2). Subgroup analyses results of PFS were as follows. In patients with type A PVTT, median PFS was 5.0 months (95\% CI: 3.3, 6.7). In patients with type B PVTT, median PFS was 14.0 months (95\% CI: $9.2,18.8)$, and in patients with type C PVTT, median PFS was 14.9 months $(95 \%$ CI: 8.5, 21.3) $(P<0.001)$ (Figure 2B and Table 2).

\section{TTP in Patients with Different Types of PVTT}

Median TTP was not yet reached of this whole cohort (Figure 3A and Table 2). Subgroup analyses results of PFS were as follows. In patients with type A PVTT, median TTP was 7.0 months (95\% CI: 1.9, 12.1). The median TTP were both not yet reached in patients with type B and type C PVTT $(P=0.016)$ (Figure 3B and Table 2).

\section{OS in Patients with Different Types of PVTT}

Of the 63 enrolled patients, 35 (55.6\%) died during followup. Disease progression was the cause of most of these deaths. Median OS was 14.8 months (95\% CI: 12.3, 17.3) for the whole population (Figure 4A and Table 2). Univariate analysis identified the following factors as affecting OS: age ( $P=0.014)$, lower AFP level $(<400 \mathrm{ng} / \mathrm{mL}, P<0.001)$, and the absence of main PVTT (PVTT type B/C, $P=0.019$ ). We then entered these significant factors in multivariate analysis by using Cox's proportional hazard model, and we found that along with AFP, the absence of main PVTT was an independent predictive factor for OS (Table 3). 
Table I Baseline Patients Characteristics

\begin{tabular}{|c|c|}
\hline Characteristics & $\begin{array}{l}\text { All Patients } \\
(n=63)\end{array}$ \\
\hline \multicolumn{2}{|l|}{ Gender } \\
\hline Male, n (\%) & $58(92.1)$ \\
\hline Female, n (\%) & $5(7.9)$ \\
\hline Age (y) & $48.7 \pm 10.5$ \\
\hline \multicolumn{2}{|l|}{ ECOG performance } \\
\hline $0, \mathrm{n}(\%)$ & $24(38.1)$ \\
\hline $\mathrm{I}, \mathrm{n}(\%)$ & $21(33.3)$ \\
\hline $2, \mathrm{n}(\%)$ & $14(22.2$ \\
\hline $3, \mathrm{n}(\%)$ & $4(6.3)$ \\
\hline \multicolumn{2}{|l|}{ Child-Pugh class } \\
\hline $\mathrm{A}, \mathrm{n}(\%)$ & $54(85.7)$ \\
\hline B, n (\%) & $9(14.3)$ \\
\hline \multicolumn{2}{|l|}{$\mathrm{HBeAg}$} \\
\hline Positive, n (\%) & $22(65.1)$ \\
\hline Negative, n (\%) & $41(34.9)$ \\
\hline WBC $\left(10^{9} / \mathrm{L}\right)$ & $5.9 \pm 2.8$ \\
\hline ALT (U/L) & $41.6 \pm 30.2$ \\
\hline AST (U/L) & $64.0 \pm 60.0$ \\
\hline PLT $\left(10^{9} / \mathrm{L}\right)$ & $173.6 \pm 89.9$ \\
\hline PT (s) & $12.7 \pm 1.2$ \\
\hline Total bilirubin level (mmol/L) & $19.2 \pm 11.3$ \\
\hline Albumin level (g/L) & $38.3 \pm 5.2$ \\
\hline \multicolumn{2}{|l|}{ a-Fetoprotein level $(\mathrm{ng} / \mathrm{mL})$} \\
\hline$\geq 400, \mathrm{n}(\%)$ & $34(54.0)$ \\
\hline$<400, \mathrm{n}(\%)$ & $29(46.0)$ \\
\hline \multicolumn{2}{|l|}{ Location of PVTT } \\
\hline $\begin{array}{l}\text { Second- or lower-order portal vein branches, } \\
\text { n (\%) }\end{array}$ & $32(50.8)$ \\
\hline First-order portal vein branch, $\mathrm{n}(\%)$ & $20(31.7)$ \\
\hline Main portal vein, $\mathrm{n}(\%)$ & II (17.5) \\
\hline \multicolumn{2}{|l|}{ Tumor number } \\
\hline Single, $n(\%)$ & $30(47.6)$ \\
\hline Multiple, n (\%) & $33(52.4)$ \\
\hline \multicolumn{2}{|l|}{ Tumor Size (cm) } \\
\hline$<7 \mathrm{~cm}, \mathrm{n}(\%)$ & $30(47.6)$ \\
\hline$\geq 7 \mathrm{~cm}, \mathrm{n}(\%)$ & $33(52.4)$ \\
\hline \multicolumn{2}{|l|}{ Prior anti-tumor therapy } \\
\hline TACE, n (\%) & $31(49.2)$ \\
\hline HAIC, n (\%) & $7(11.1)$ \\
\hline Sorafenib, n (\%) & $8(12.7)$ \\
\hline Lenvatinib, n (\%) & $6(9.5)$ \\
\hline unknown, n (\%) & II (I7.5) \\
\hline Mean follow up (months) & $12.6 \pm 4.5$ \\
\hline
\end{tabular}

Abbreviations: ECOG, Eastern Cooperative Oncology Group; WBC, white blood cell; ALT, alanine aminotransferase; AST, aspartate aminotransferase; PLT, platelet count; PT, prothrombin time; TBIL, total bilirubin; ALB, albumin; AFP, $\alpha$ fetoprotein; PVTT, portal vein tumor thrombus; TACE, transarterial chemoembolization; HAIC, hepatic arterial infusion chemotherapy.
Subgroup analyses (Figure 4B and Table 2) of OS in patients with different types of PVTT showed that the median OS of patients with type A, B, or C PVTT was 5.8 months (95\% CI: $3.9,7.7), 15.9$ months (95\% CI: 12.1 , 19.7), and 16.0 months (95\% CI: $13.7,18.3)$, respectively $(P<0.001)$. The 0.5 -year OS rate for the whole population was $87.3 \%$, and was $45.5 \%, 95.0 \%$, and $96.9 \%$ for patients with type A, B, and C PVTT. The 1-year OS rate for the whole population was $60.0 \%$, and was $27.3 \%, 65.0 \%$, and $68.8 \%$ for patients with type A, B, and C PVTT.

\section{Liver Reserve Function}

At baseline, 54 patients were in Child-Pugh (CP) score $\mathrm{A}$ and 9 patients in $\mathrm{CP}$ score $\mathrm{B}$. At the end of this study, 24 patients were in $\mathrm{CP}$ score $\mathrm{A}, 21$ patients in $\mathrm{CP}$ score $\mathrm{B}$, and 18 in $\mathrm{CP}$ score $\mathrm{C}$ (data un-presented). Among the 18 patients in $\mathrm{CP}$ score $\mathrm{C}, 13$ died of liver failure at last. None of the 13 deaths were caused by treatment-related adverse events (TRAEs) after discussion by the multidisciplinary team (MDT).

\section{Safety Analysis}

All recorded TRAEs are shown in Table 4. Thirty-one patients $(31 / 63,49.2 \%)$ experienced at least one adverse event (AE). Overall, the most frequent AEs were hand-foot skin reaction $(33 / 63,52.4 \%)$, abdominal pain $(31 / 63,49.2 \%)$, hepatitis $(29 / 63,46.0 \%)$, and thrombocytopenia (27/63, $42.9 \%$ ) for all included patients. Grade 3/4 AEs occurred in 18 patients $(28.6 \%)$, and finally led to treatment interruption in 11 patients. No one died of TRAE in this study.

\section{Discussion}

Poor outcomes for advanced HCC remain a great challenge, especially for patients with PVTT, as PVTT may cause extensive intrahepatic spread and portal hypertension, resulting in a high degree of malignancy and treatment difficulties. ${ }^{32,33}$ The recommended treatment strategies for PVTT patients are sorafenib and TACE according to BCLC. ${ }^{8}$ However, both therapies remained unsatisfactory in our daily clinical application. Currently, a series of clinical trials have shown that anti-PD-1 monotherapy is effective in treating advanced HCC patients with an ORR of $10-20 \%{ }^{34,35}$ Indeed, the IMbrave150 study has demonstrated a new regimen to be superior to sorafenib for improving median OS in advanced HCC. ${ }^{36}$ However, there is no study available now analyzing the efficacy and safety of PD-1-based therapy for HCC patients with PVTT. Herein, we conduct a multi-center treatment cohort of HBV 
Table 2 Tumor Responses and Survival in Patients with Different Types of PVTT

\begin{tabular}{|c|c|c|c|c|}
\hline & All Patients $N=63$ & Type A $n=I I$ & Type B $n=20$ & Type C n=32 \\
\hline \multicolumn{5}{|l|}{ Tumor response } \\
\hline Complete response $(\mathrm{CR})$ & I (I.6\%) & 0 & I (5.0\%) & 0 \\
\hline Partial response (PR) & $27(42.8 \%)$ & $3(27.3 \%)$ & $9(45.0 \%)$ & $15(46.9 \%)$ \\
\hline Stable disease (SD) & $19(30.2 \%)$ & $3(27.3 \%)$ & $9(45.0 \%)$ & 7 (21.9\%) \\
\hline Progressive disease (PD) & $16(25.4 \%)$ & $5(45.4 \%)$ & I (5.0\%) & $10(31.2 \%)$ \\
\hline ORR $(C R+P R)^{*}$ & $28(44.0 \%)$ & $3(27.3 \%)$ & $10(50.0 \%)$ & $15(46.9 \%)$ \\
\hline $\mathrm{DCR}(\mathrm{CR}+\mathrm{PR}+\mathrm{SD})^{\#}$ & $47(75.0 \%)$ & $6(54.5 \%)$ & $19(95.0 \%)$ & $22(68.8 \%)$ \\
\hline \multicolumn{5}{|l|}{ Survival (months) } \\
\hline PFS median $(95 \% \mathrm{Cl})$ & II.8 (6.4-17.2) & $5.0(3.3-6.7)$ & $14.0(9.2-18.8)$ & $\mid 4.9(8.5-2 \mid .3)$ \\
\hline TTP median $(95 \% \mathrm{Cl})$ & NR & $7.0(1.9-12.1)$ & NR & NR \\
\hline OS median $(95 \% \mathrm{Cl})$ & $14.8(12.3-17.3)$ & $5.8(3.9-7.7)$ & $15.9(12.1-19.7)$ & $16.0(13.7-18.3)$ \\
\hline 6 months OS\% & $87.3 \%$ & $45.5 \%$ & $95.0 \%$ & $96.9 \%$ \\
\hline 12 months OS\% & $60.0 \%$ & $27.3 \%$ & $65.0 \%$ & $68.8 \%$ \\
\hline
\end{tabular}

Notes: *Pearson $\chi 2=1.640, P=0.440$ (for all patients). ${ }^{*}$ Pearson $\chi 2=7.306, P=0.026$ (for all patients).

Abbreviations: CR, complete response; PR, partial response; SD, stable disease; PD, progressive disease; ORR, objective response rate; DCR, disease control rate; PFS, progression-free survival; TTP, time to tumor progression; OS, overall survival; NR, not reached.

associated HCC patients with PVTT in China and found that: 1) treatment with camrelizumab-apatinib appears to have better survival benefits compared with type $\mathrm{A}$ in patients with HCC and PVTT; 2) along with AFP, the absence of main PVTT was an independent predictive factor for OS; 3. "C $+\mathrm{A}$ " therapy was well tolerated generally and had manageable side effects.

A large phase I/II study of nivolumab (CheckMate040) including 262 advanced HCC patients with or without previous exposure to sorafenib showed an ORR of $18 \%$ and a promising 1 -year OS rate of $62 \%{ }^{34}$ Another open-label, no-randomized Phase II study (KEYNOTE-
224), pembrolizumab treatment has demonstrated an ORR of $16.3 \%$ and $77.9 \%$ 6-month OS. ${ }^{35}$ Similar in a randomized phase II trial of camrelizumab including 217 HCC patients showed an ORR of $13.8 \%$ and $74.7 \%$ 6-month OS. ${ }^{20}$ By contrast, our current study showed a higher ORR of $44.0 \%$ and a promising 6-month OS rate of $87.3 \%$. Importantly, most of the included patients in our current study were previously treated. In line with our results, a Phase $1 \mathrm{~b}$ study of atezolizumab plus bevacizumab (GO30140, NCT02715531) has also demonstrated that antiangiogenic agents and checkpoint inhibitors combination therapy have synergistic anti-tumor activity in
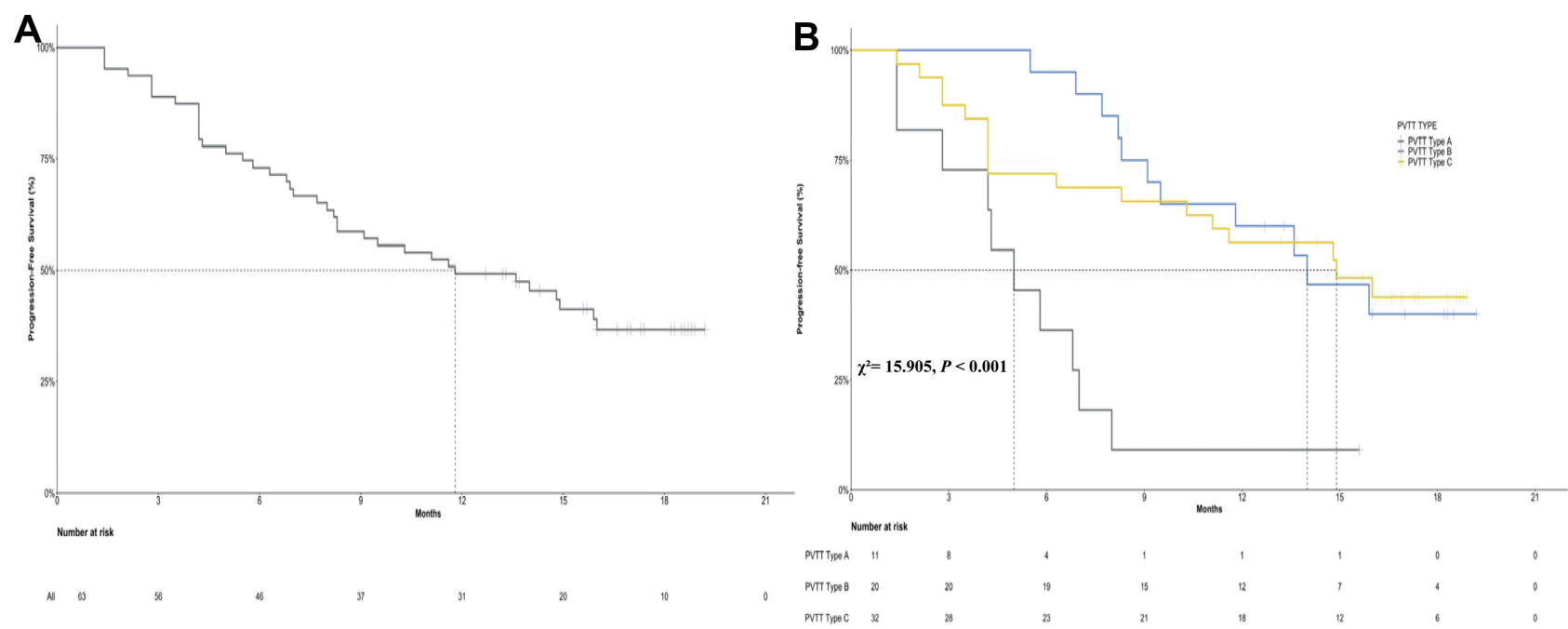

Figure 2 Kaplan-Meier curve of PFS in patients with HCC and PVTT who underwent "C + A" therapy: (A) for the whole cohort of patients; (B) for patients with different types of PVTT. 

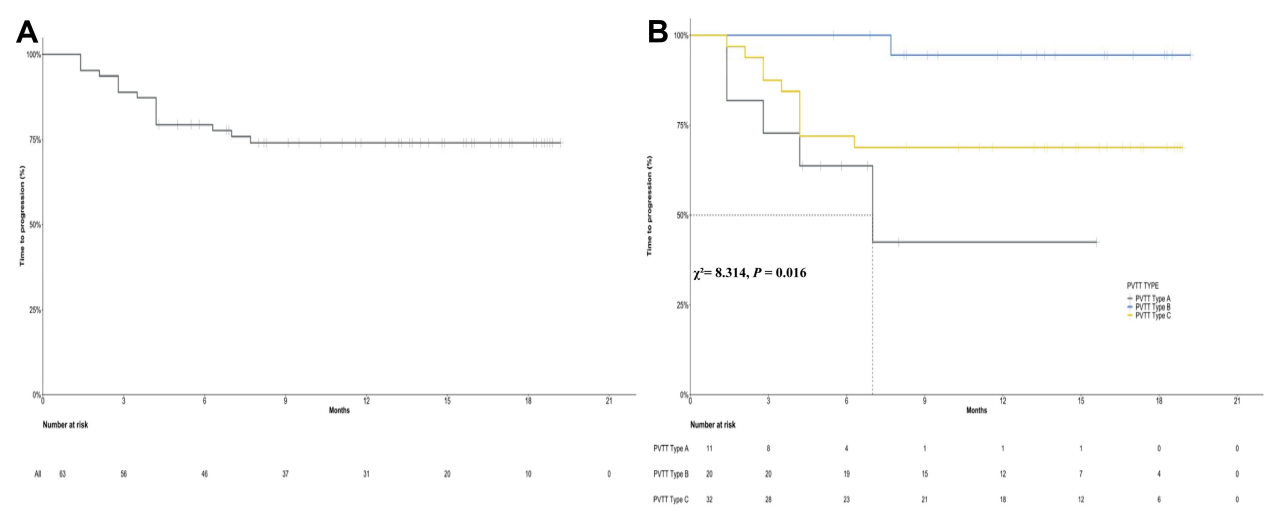

Figure 3 Kaplan-Meier curve of TTP in patients with HCC and PVTT who underwent " $\mathrm{C}+\mathrm{A}$ " therapy: (A) for the whole cohort of patients; (B) for patients with different types of PVTT.

A

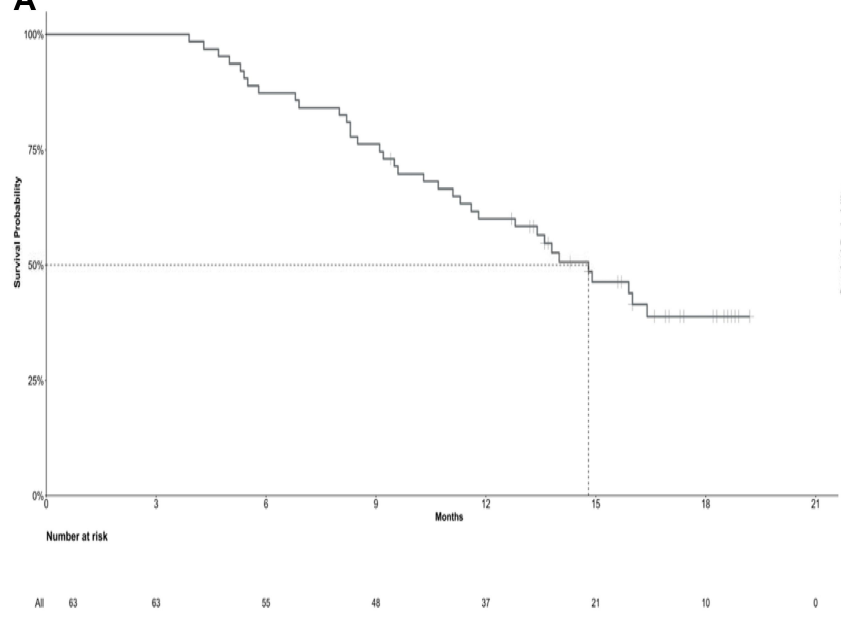

B

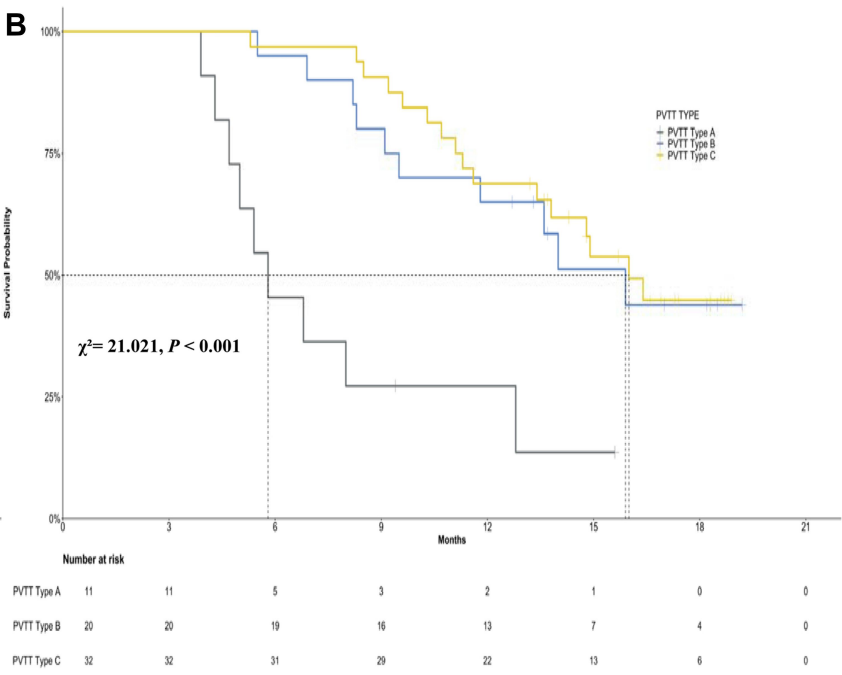

Figure 4 Kaplan-Meier curve of OS in patients with HCC and PVTT who underwent "C + A" therapy: (A) for the whole cohort of patients; (B) for patients with different types of PVTT.

advanced HCC. ${ }^{21,22,36}$ In IMbrave150 study, the median OS was not reached in the atezolizumab plus bevacizumab arm and was 13.2 months (95\% CI, 10.4 to not reached) in the sorafenib arm. The OS rates at 6 months were $84.8 \%$ and $72.2 \%$ in the atezolizumab plus bevacizumab and sorafenib arm, respectively. Furthermore, the combination of camrelizumab and apatinib has also been examined in phase I and II study showing an ORR of $50 \% .{ }^{26}$ Besides, lenvatinib plus pembrolizumab also showed strong antitumor activity in patients with unresectable HCC, with a 46\% ORR, a median PFS of 9.7 months, and a median OS of 20.4 months. $^{37}$ One of the possible drives to a potentially higher than anti-PD-1 monotherapy is that anti-VEGF therapies can reduce VEGF-mediated immunosuppression within the tumor and its microenvironment, and may enhance anti-PD-1/PD-L1 efficacy by reversing
VEGF-mediated immunosuppression and promoting tumor T-cell infiltration. ${ }^{38}$ Comparing with previous studies analyzing the combination of anti-PD-1/PD-L1 and antiangiogenesis therapy, there are two main advantages of the " $\mathrm{C}+\mathrm{A}$ " regimen: 1 ) bevacizumab is a monoclonal antibody and needs intravenous infusion, while apatinib only needs oral administration, which is more convenient for patients; 2) in the IMbrave 150 trial, the incidence of upper gastrointestinal bleeding observed in the atezolizumab-bevacizumab group was $7 \%$ since bleeding is a known adverse event to bevacizumab. In contrast, our study shown that only $2(3.2 \%)$ patients have upper gastrointestinal bleeding during " $\mathrm{C}+\mathrm{A}$ " therapy.

Another important finding in this study was that PVTT involving the main portal vein was an independent predictive factor for OS. And patients with PVTT in the first-order 
Table 3 Univariate and Multivariate Analyses of Baseline Variables Affecting OS

\begin{tabular}{|c|c|c|c|c|c|c|}
\hline & \multicolumn{3}{|c|}{ Univariate } & \multicolumn{3}{|c|}{ Multivariate } \\
\hline & HR & $95 \% \mathrm{Cl}$ & $P$ value & HR & $95 \% \mathrm{Cl}$ & $P$ value \\
\hline Gender: F/M & 0.087 & $0.003-2.810$ & 0.168 & & & \\
\hline Age & 0.942 & $0.898-0.988$ & 0.014 & 0.972 & $0.940-1.006$ & 0.104 \\
\hline ECOG performance: & & & 0.166 & & & \\
\hline $0 / 3$ & 1.423 & $0.191-10.602$ & 0.730 & & & \\
\hline $1 / 3$ & 4.566 & $0.609-34.238$ & 0.140 & & & \\
\hline $2 / 3$ & 1.515 & $0.237-9.735$ & 0.660 & & & \\
\hline Child-Pugh class: $A / B$ & 0.398 & $0.106-1.489$ & 0.171 & & & \\
\hline HBeAg: N/P & 0.486 & $0.189-1.252$ & 0.135 & & & \\
\hline WBC & 1.003 & $0.846-1.190$ & 0.969 & & & \\
\hline ALT & 1.004 & $0.983-1.025$ & 0.722 & & & \\
\hline AST & 0.999 & $0.988-1.010$ & 0.896 & & & \\
\hline PLT & 0.999 & $0.993-1.006$ & 0.835 & & & \\
\hline PT & 0.898 & $0.563-1.434$ & 0.653 & & & \\
\hline TBIL & 1.032 & $0.985-1.082$ & 0.185 & & & \\
\hline ALB & 1.002 & $0.910-1.103$ & 0.966 & & & \\
\hline AFP level: $<400 / \geq 400$ & 0.051 & $0.014-0.189$ & 0.000 & 0.146 & $0.059-0.361$ & 0.000 \\
\hline Type of PVTT: & & & 0.019 & & & 0.005 \\
\hline $\mathrm{B} / \mathrm{A}$ & 0.144 & $0.032-0.649$ & 0.012 & 0.278 & $0.113-0.687$ & 0.005 \\
\hline $\mathrm{C} / \mathrm{A}$ & 0.122 & $0.027-0.563$ & 0.007 & 0.218 & $0.083-0.573$ & 0.002 \\
\hline Tumor number: Single/Multiple & 0.605 & $0.235-1.558$ & 0.298 & & & \\
\hline Tumor Size: $<7 \mathrm{~cm} / \geq 7 \mathrm{~cm}$ & 0.691 & $0.290-1.645$ & 0.404 & & & \\
\hline Prior anti-tumor therapy & & & 0.512 & & & \\
\hline HAIC/TACE & 1.213 & $0.675-7.332$ & 0.536 & & & \\
\hline Sorafenib/TACE & 1.733 & $0.221-11.314$ & 0.765 & & & \\
\hline Lenvatinib/TACE & 0.878 & $0.467-9.944$ & 0.251 & & & \\
\hline unknown/TACE & 1.632 & $0.654-6.377$ & 0.911 & & & \\
\hline
\end{tabular}

Abbreviations: OS, overall survival; ECOG, Eastern Cooperative Oncology Group; WBC, white blood cell; ALT, alanine aminotransferase; AST, aspartate aminotransferase; PLT, platelet count; PT, prothrombin time; TBIL, total bilirubin; ALB, albumin; AFP, $\alpha$-fetoprotein; PVTT, portal vein tumor thrombus; TACE, transarterial chemoembolization; HAIC, hepatic arterial infusion chemotherapy.

portal vein branch or in the second- or lower-order portal vein branches had better outcome. A likely mechanism is that, as a highly selective VEGFR-2 blocker, apatinib can effectively inhibit VEGF-stimulated endothelial cell migration and proliferation, which is required for native collateral formation and arteriogenesis. ${ }^{39-41}$ Consistent with the previous TACE plus apatinib or TACE plus sorafenib studies in patients with HCC and PVTT, ${ }^{13,15,23,25,42}$ our data suggest that regimen with better effectiveness is urgently needed for patients with main PVTT.

In the current study, median TTPs were not reached for PVTT type B and C, while median PFSs were reached. By analyzing the data, we notice that a total of 22 patients died before $\mathrm{PD}$, among whom, 13 died of worsening in liver function, 3 died of respiratory failure, 3 died of heart failure,
2 died of uremia, 1 died of acute cerebral infarction and no one died of treatment-associated AEs (data un-presented).

Importantly, our data also demonstrated that " $\mathrm{C}+\mathrm{A}$ " regimen were well tolerated generally and had manageable side effects, with the most common drug-related adverse events being Hand-foot skin reaction, abdominal pain, hepatitis and thrombocytopenia, which were similar to previous reports. ${ }^{20,26}$ Most adverse events in the current study were grade 1 or 2 and were well tolerated by patients without the need for dose reduction or suspension of medication. These symptoms were gradually alleviated and disappeared within 1 or 2 weeks. Besides, we did not record any reactive cutaneous capillary endothelial proliferation (RCCEP) in our cohort, which was the most common camrelizumab-related $\mathrm{AE}$ in camrelizumab 
Table 4 Treatment Related Adverse Events

\begin{tabular}{|c|c|c|c|c|c|}
\hline Adverse Event & $\begin{array}{l}\text { All Grades, n (\%), } \\
\mathrm{N}=63\end{array}$ & $\begin{array}{l}\text { Grade } 3 / 4, \text { n (\%), } \\
N=63\end{array}$ & $\begin{array}{l}\text { Type A, n (\%), } \\
\text { N=I I }\end{array}$ & $\begin{array}{l}\text { Type B, n (\%), } \\
\text { N=20 }\end{array}$ & $\begin{array}{l}\text { Type C, n (\%), } \\
\text { N=32 }\end{array}$ \\
\hline Diarrhea & $19(30.2)$ & $2(3.2)$ & $4(36.4)$ & $6(30.0)$ & $9(28.1)$ \\
\hline Headache & $17(27.0)$ & I (I.6) & $4(36.4)$ & $7(35.0)$ & $6(18.8)$ \\
\hline Abdominal pain & $31(49.2)$ & $3(4.8)$ & $7(63.6)$ & $13(65.0)$ & II (34.4) \\
\hline Cough & $16(25.4)$ & 0 & $3(27.3)$ & $7(35.0)$ & $6(18.8)$ \\
\hline Fatigue & $14(22.2)$ & 0 & $5(45.5)$ & $3(15.0)$ & $6(18.8)$ \\
\hline Vomiting & $7(I I . I)$ & 0 & I (9.I) & $3(15.0)$ & $3(9.4)$ \\
\hline Hand-foot skin reaction & $33(52.4)$ & $2(3.2)$ & $8(72.7)$ & $9(45.0)$ & $16(50.0)$ \\
\hline Hepatitis* & $29(46.0)$ & $3(4.8)$ & $7(63.6)$ & $12(60.0)$ & $10(31.3)$ \\
\hline Thrombocytopenia & $27(42.9)$ & I (I.6) & $4(36.4)$ & $9(45.0)$ & $14(43.8)$ \\
\hline Leukopenia & $16(25.4)$ & 0 & $5(45.5)$ & $3(15.0)$ & $8(25.0)$ \\
\hline Hypothyroidism & $22(34.9)$ & $3(4.8)$ & $3(27.3)$ & $9(45.0)$ & $10(31.3)$ \\
\hline Hypertension & $13(20.6)$ & 0 & $6(54.5)$ & $2(10.0)$ & $5(15.6)$ \\
\hline Proteinuria & $21(33.3)$ & $I(1.6)$ & $4(36.4)$ & $8(40.0)$ & $9(28.1)$ \\
\hline Dental ulcer & $5(7.9)$ & 0 & 0 & $3(15.0)$ & $2(6.3)$ \\
\hline Gingival bleeding & $4(6.3)$ & 0 & I (9.1) & $3(15.0)$ & 0 \\
\hline Hoarseness & $2(3.2)$ & 0 & I (9.I) & 0 & I (3.I) \\
\hline $\begin{array}{l}\text { Gastrointestinal } \\
\text { hemorrhage }\end{array}$ & $2(3.2)$ & 0 & 0 & I (5.0) & I (3.I) \\
\hline Rash & $19(30.2)$ & $2(3.2)$ & $5(45.5)$ & $4(20.0)$ & $10(31.3)$ \\
\hline
\end{tabular}

Notes: Data are numbers of events. Data in parentheses are percentages. There were no grade 5 adverse events in all patients; *Alanine aminotransferase (ALT) or aspartate aminotransferase (AST) increased.

monotherapy, ${ }^{20}$ indicating that apatinib combination therapy might reduce the occurrence of some camrelizumabrelated AEs or SAEs.

There are some limitations in our study. First, this study was retrospectively designed and the sample size is relatively small, though objective endpoints (especially imaging data for tumor responses assessment) were elaborately and integrally recorded. Second, the mean duration of follow-up was not long enough (the mean duration of follow-up was $12.6 \pm 4.5$ months) as we have not applied camrelizumab for HCC patients until 2018. But we believe the above data provide important experience on subgroups of patients frequently found in our everyday clinical practice and MDT consultation. Third, 31 patients were excluded from the final analysis, which might reduce the power of statistical analysis. But despite all that, the strength of our study is the provision of unique data on the safety and efficacy of camrelizumab and apatinib combination therapy, which might be the first report to evaluate PD-1 blocker and selective VEGFR-2 blocker for HCC patients with PVTT.

In conclusion, our findings in this study support further applications of camrelizumab and apatinib combination therapy in HCC patients. As non-randomized retrospective observational data, this study can only provide limited evidence to show that these drugs are efficacious and safe. It shall not be viewed as non-biased data and utilized in clinical decision-making without further evidence base. What our study suggests is that camrelizumab and apatinib combination therapy appeared to have a promising outcome and manageable side effects in treating advanced HCC with PVTT, especially for PVTT in the first-order portal vein branch or in the second- or lower-order portal vein branches, which is worth of popularization and application in clinical practice. However, better prospective designed studies in the future are needed to have a longer follow-up, larger research samples and different strategy combinations.

\section{Data Sharing Statement}

The data that support the findings of this study are available from the corresponding author upon reasonable request.

\section{Statement of Ethics}

This study was designed and performed according to the Helsinki Declaration and was approved by the Medical Ethics Committee of every participating hospitals in this study (NFEC-2019-069, no. 2019-072, SDEC-2019-011, ZJEC-2019-122, and HNEC-2019-024), and written informed consent was obtained from each patient to retroactively review and report on their medical records. 


\section{Acknowledgments}

This study was partly supported by the grants from the Natural Science Foundation of Guangdong Province (2017A030313645 and 2018A030313398), Self Financing Science and Technology Project of Foshan City (2018AB00963) and WBE Liver Fibrosis Foundation (CFHPC2020031). The funding agencies had no role in study design, data collection and analysis, decision to publish, or preparation of the manuscript.

\section{Author Contributions}

All authors made a significant contribution to the work reported, whether that is in the conception, study design, execution, acquisition of data, analysis and interpretation, or in all these areas; took part in drafting, revising or critically reviewing the article; gave final approval of the version to be published; have agreed on the journal to which the article has been submitted; and agree to be accountable for all aspects of the work.

\section{Disclosure}

The authors report no potential conflicts of interest for this work.

\section{References}

1. Bray F, Ferlay J, Soerjomataram I, Siegel RL, Torre LA, Jemal A. Global cancer statistics 2018: GLOBOCAN estimates of incidence and mortality worldwide for 36 cancers in 185 countries. CA Cancer J Clin. 2018;68:394-424. doi:10.3322/caac.21492

2. Yang JD, Hainaut P, Gores GJ, Amadou A, Plymoth A, Roberts LR. A global view of hepatocellular carcinoma: trends, risk, prevention and management. Nat Rev Gastroenterol Hepatol. 2019;16:589-604. doi:10.1038/s41575-019-0186-y

3. Vogel A, Cervantes A, Chau I, et al. Hepatocellular carcinoma: ESMO clinical practice guidelines for diagnosis, treatment and follow-up. Ann Oncol. 2019;30:871-873. doi:10.1093/annonc/mdy510

4. Galle PR, Forner A, Llovet JM; EASL Clinical Practice. Guidelines: management of hepatocellular carcinoma. $J$ Hepatol. 2018;69:182-236. doi:10.1016/j.jhep.2018.03.019

5. Heimbach JK, Kulik LM, Finn RS, et al. AASLD guidelines for the treatment of hepatocellular carcinoma. Hepatology. 2018;67:358-380.

6. Novi M, Lauritano EC, Piscaglia AC, et al. Portal vein tumor thrombosis revascularization during sorafenib treatment for hepatocellular carcinoma. Am J Gastroenterol. 2009;104:1852-1854. doi:10.1038/ajg.2009.140

7. Liu PH, Huo TI, Miksad RA. Hepatocellular carcinoma with portal vein tumor involvement: best management strategies. Semin Liver Dis. 2018;38:242-251. doi:10.1055/s-0038-1666805

8. Cheng S, Chen M, Cai J, et al. Chinese expert consensus on multidisciplinary diagnosis and treatment of hepatocellular carcinoma with portal vein tumor thrombus (2018 Edition). Liver Cancer. 2020;9:28-40. doi:10.1159/000503685

9. Zhang XP, Wang K, Li N, et al. Survival benefit of hepatic resection versus transarterial chemoembolization for hepatocellular carcinoma with portal vein tumor thrombus: a systematic review and meta-analysis. Bmc Cancer. 2017;17:902. doi:10.1186/s12885-017-3895-z
10. Huo YR, Eslick GD. Transcatheter arterial chemoembolization plus radiotherapy compared with chemoembolization alone for hepatocellular carcinoma: a systematic review and meta-analysis. JAMA Oncol. 2015;1:756-765. doi:10.1001/jamaoncol.2015.2189

11. Luo J, Guo RP, Lai EC, et al. Transarterial chemoembolization for unresectable hepatocellular carcinoma with portal vein tumor thrombosis: a prospective comparative study. Ann Surg Oncol. 2011;18:413-420. doi:10.1245/s10434-010-1321-8

12. Xue TC, Xie XY, Zhang L, Yin X, Zhang BH, Ren ZG. Transarterial chemoembolization for hepatocellular carcinoma with portal vein tumor thrombus: a meta-analysis. BMC Gastroenterol. 2013;13:60. doi:10.1186/1471-230X-13-60

13. Zhang X, Wang K, Wang M, et al. Transarterial chemoembolization (TACE) combined with sorafenib versus TACE for hepatocellular carcinoma with portal vein tumor thrombus: a systematic review and meta-analysis. Oncotarget. 2017;8:29416-29427. doi:10.18632/ oncotarget.15075

14. Kuo YH, Wu IP, Wang JH, et al. The outcome of sorafenib monotherapy on hepatocellular carcinoma with portal vein tumor thrombosis. Invest New Drugs. 2018;36:307-314. doi:10.1007/ s10637-017-0468-6

15. Zhang Y, Fan W, Wang Y, et al. Sorafenib with and without transarterial chemoembolization for advanced hepatocellular carcinoma with main portal vein tumor thrombosis: a retrospective analysis. Oncologist. 2015;20:1417-1424. doi:10.1634/theoncologist.2015-0196

16. Rimassa L, Pressiani T, Merle P. Systemic treatment options in hepatocellular carcinoma. Liver Cancer. 2019;8:427-446. doi:10.1159/000499765

17. Finn RS, Zhu AX, Farah W, et al. Therapies for advanced stage hepatocellular carcinoma with macrovascular invasion or metastatic disease: A systematic review and meta-analysis. Hepatology. 2018;67:422-435. doi:10.1002/hep.29486

18. Finn RS, Zhu AX. Evolution of systemic therapy for hepatocellular carcinoma. Hepatology. 2020 May 7. doi:10.1002/hep.31306

19. Brown ZJ, Greten TF, Heinrich B. Adjuvant treatment of hepatocellular carcinoma: prospect of immunotherapy. Hepatology. 2019;70:1437-1442. doi:10.1002/hep.30633

20. Markham A, Keam SJ. Camrelizumab: first global approval. Drugs. 2019;79:1355-1361. doi:10.1007/s40265-019-01167-0

21. Lee MS, Ryoo BY, Hsu CH, et al. Atezolizumab with or without bevacizumab in unresectable hepatocellular carcinoma (GO30140): an open-label, multicentre, phase $1 \mathrm{~b}$ study. Lancet Oncol. 2020;21:808-820. doi:10.1016/S1470-2045(20)30156-X

22. Finn RS, Qin S, Ikeda M, et al. Atezolizumab plus bevacizumab in unresectable hepatocellular carcinoma. $N$ Engl $J$ Med. 2020;382:1894-1905. doi:10.1056/NEJMoa1915745

23. Liu C, Xing W, Si T, Yu H, Guo Z. Efficacy and safety of apatinib combined with transarterial chemoembolization for hepatocellular carcinoma with portal venous tumor thrombus: a retrospective study. Oncotarget. 2017;8:100734-100745. doi:10.18632/ oncotarget.20140

24. Lu W, Jin XL, Yang C, et al. Comparison of efficacy between TACE combined with apatinib and TACE alone in the treatment of intermediate and advanced hepatocellular carcinoma: A single-center randomized controlled trial. Cancer Biol Ther. 2017;18:433-438. doi:10.1080/15384047.2017.1323589

25. Fan W, Yuan G, Fan H, et al. Apatinib combined with transarterial chemoembolization in patients with hepatocellular carcinoma and portal vein tumor thrombus: a multicenter retrospective study. Clin Ther. 2019;41:1463-1476. doi:10.1016/j.clinthera.2019.04.036

26. Xu J, Zhang Y, Jia R, et al. Anti-PD-1 Antibody SHR-1210 combined with apatinib for advanced hepatocellular carcinoma, gastric, or esophagogastric junction cancer: an open-label, dose escalation and expansion study. Clin Cancer Res. 2019;25:515-523. doi:10.1158/ 1078-0432.CCR-18-2484 
27. Llovet JM, Bru C, Bruix J. Prognosis of hepatocellular carcinoma: the BCLC staging classification. Semin Liver Dis. 1999;19:329-338. doi:10.1055/s-2007-1007122

28. Kodama K, Kawaoka T, Aikata H, et al. Comparison of outcome of hepatic arterial infusion chemotherapy combined with radiotherapy and sorafenib for advanced hepatocellular carcinoma patients with major portal vein tumor thrombosis. Oncology. 2018;94:215-222. doi:10.1159/000486483

29. Zhang YF, Shang H, Zeng XL, Ji H, Li YM, Lu HW. Postoperative adjuvant chemo (embolization) therapy for hepatocellular carcinoma with portal vein tumor thrombosis. Onco Targets Ther. 2018;11:5407-5417. doi:10.2147/OTT.S171612

30. Mizandari M, Azrumelashvili T, Paksashvili N, et al. Tumor regression in HCC patient with portal vein tumor thrombosis after intraportal radiofrequency thermal ablation. Case Reports Hepatol. 2016;2016:6843121.

31. Lencioni R, Llovet JM. Modified RECIST (mRECIST) assessment for hepatocellular carcinoma. Semin Liver Dis. 2010;30:52-60. doi:10.1055/s-0030-1247132

32. Wang JC, Xia AL, Xu Y, Lu XJ. Comprehensive treatments for hepatocellular carcinoma with portal vein tumor thrombosis. J Cell Physiol. 2019;234:1062-1070. doi:10.1002/jcp.27324

33. Chan SL, Chong CC, Chan AW, Poon DM, Chok KS. Management of hepatocellular carcinoma with portal vein tumor thrombosis review and update at 2016. World $J$ Gastroenterol. 2016;22:7289-7300. doi:10.3748/wjg.v22.i32.7289

34. El-Khoueiry AB, Sangro B, Yau T, et al. Nivolumab in patients with advanced hepatocellular carcinoma (CheckMate 040): an open-label, non-comparative, phase $1 / 2$ dose escalation and expansion trial. Lancet. 2017;389:2492-2502. doi:10.1016/S0140-6736(17)31046-2
35. Zhu AX, Finn RS, Edeline J, et al. Pembrolizumab in patients with advanced hepatocellular carcinoma previously treated with sorafenib (KEYNOTE-224): a non-randomised, open-label phase 2 trial. Lancet Oncol. 2018;19:940-952. doi:10.1016/S1470-2045(18)30351-6

36. Kelley RK. Atezolizumab plus bevacizumab - a landmark in liver cancer. $N$ Engl J Med. 2020;382:1953-1955. doi:10.1056/ NEJMe2004851

37. Finn RS, Ikeda M, Zhu AX, et al. Phase ib study of lenvatinib plus pembrolizumab in patients with unresectable hepatocellular carcinoma. J Clin Oncol. 2020;O2000808.

38. Hegde PS, Wallin JJ, Mancao C. Predictive markers of anti-VEGF and emerging role of angiogenesis inhibitors as immunotherapeutics Semin Cancer Biol. 2018;52:117-124. doi:10.1016/j.semcancer. 2017.12.002

39. Lan CY, Wang Y, Xiong Y, et al. Apatinib combined with oral etoposide in patients with platinum-resistant or platinum-refractory ovarian cancer (AEROC): a phase 2, single-arm, prospective study. Lancet Oncol. 2018;19:1239-1246. doi:10.1016/S1470-2045(18) 30349-8

40. Li J, Qin S, Xu J, et al. Apatinib for chemotherapy-refractory advanced metastatic gastric cancer: results from a randomized, placebo-controlled, parallel-arm, phase II trial. $J$ Clin Oncol. 2013;31:3219-3225. doi:10.1200/JCO.2013.48.8585

41. Zhang S. Problematic analysis and inadequate toxicity data in phase iii apatinib trial in gastric cancer. J Clin Oncol. 2016;34:3821. doi: 10.1200/JCO.2016.67.3889

42. Pan T, Li XS, Xie QK, et al. Safety and efficacy of transarterial chemoembolization plus sorafenib for hepatocellular carcinoma with portal venous tumour thrombus. Clin Radiol. 2014;69:e553-61. doi:10.1016/j.crad.2014.09.007
OncoTargets and Therapy

\section{Publish your work in this journal}

OncoTargets and Therapy is an international, peer-reviewed, open access journal focusing on the pathological basis of all cancers, potential targets for therapy and treatment protocols employed to improve the management of cancer patients. The journal also focuses on the impact of management programs and new therapeutic

\section{Dovepress}

agents and protocols on patient perspectives such as quality of life, adherence and satisfaction. The manuscript management system is completely online and includes a very quick and fair peer-review system, which is all easy to use. Visit http://www.dovepress.com/ testimonials.php to read real quotes from published authors. 